\title{
Dynamic Optical Coherence Tomography in Dermatology
}

\author{
Martina Ulrich ${ }^{a}$ Lotte Themstrup $^{c}$ Nathalie de Carvalho ${ }^{d}$ Marco Manfredi ${ }^{d}$ \\ Costantino Granad Silvana Ciardo $^{d}$ Raphaela Kästle $^{b}$ Jon Holmes ${ }^{e}$ \\ Richard Whitehead $^{\mathrm{e}}$ Gregor B.E. Jemec $^{c}$ Giovanni Pellacani $^{\mathrm{d}}$ Julia Welzel ${ }^{\mathrm{b}}$ \\ ${ }^{a}$ CMB Collegium Medicum Berlin, Berlin, and ${ }^{b}$ Department of Dermatology, General Hospital Augsburg, Augsburg, \\ Germany; ' ${ }^{\mathrm{D}}$ Department of Dermatology, University of Copenhagen, Roskilde Hospital, Roskilde, Denmark; \\ ${ }^{\mathrm{d}}$ Department of Dermatology, University of Modena and Reggio Emilia, Modena, Italy; ${ }^{\mathrm{e} M i c h e l s o n ~ D i a g n o s t i c s,}$ \\ Maidstone, UK
}

\section{Key Words}

Optical coherence tomography - Skin cancer - Diagnosis .

Basal cell carcinoma - Squamous cell carcinoma - Actinic

keratosis $\cdot$ Melanoma $\cdot$ Vascularity $\cdot$ Prognosis

\begin{abstract}
Optical coherence tomography (OCT) represents a non-invasive imaging technology, which may be applied to the diagnosis of non-melanoma skin cancer and which has recently been shown to improve the diagnostic accuracy of basal cell carcinoma. Technical developments of OCT continue to expand the applicability of OCT for different neoplastic and inflammatory skin diseases. Of these, dynamic OCT (D-OCT) based on speckle variance OCT is of special interest as it allows the in vivo evaluation of blood vessels and their distribution within specific lesions, providing additional functional information and consequently greater density of data. In an effort to assess the potential of D-OCT for future scientific and clinical studies, we have therefore reviewed the literature and preliminary unpublished data on the visualization of the microvasculature using D-OCT. Information on D-OCT in skin cancers including melanoma, as well as in a variety of
\end{abstract}

other skin diseases, is presented in an atlas. Possible diagnostic features are suggested, although these require additional validation.

(c) 2016 S. Karger AG, Basel

\section{Introduction}

Optical coherence tomography (OCT) is a non-invasive imaging technology, which allows the evaluation of the skin at high resolution and with no discernible effect on the tissue. OCT is a well-established diagnostic tool in ophthalmology, but technical limitations have previously restricted its widespread use in dermatology. OCT was first applied to skin imaging in 1997 [1], and the early images demonstrated the potential to identify skin structures such as the dermal-epidermal junction (DEJ) and sweat ducts. Whereas these early studies were unable to reliably identify non-melanocytic skin tumours [2], recent improvements in technology including multi-beam OCT have helped to overcome these limitations [3-6]. Technological developments have led to an increased resolution, a larger field of view as well as an increased scanning speed 
in current systems. Furthermore, the combination of the classical vertical OCT images and horizontal view is now established routine, and adds additional value to the diagnostic capability of the technique. Thus, a more detailed analysis of skin structures is now possible, leading to an improvement of image quality and diagnostic capability.

In consequence of these improvements, OCT criteria for the diagnosis of basal cell carcinoma (BCC) have now been described. When added in the clinical diagnostic workflow of BCC, the additional use of OCT improved the diagnostic accuracy to $87.4 \%$. In comparison, diagnostic accuracy for clinical assessment was 65.8 and $76.2 \%$ for dermoscopy, respectively [7].

In the current clinical workflow, OCT is mainly used for diagnosis and monitoring of non-melanoma skin cancer (NMSC) including mainly BCC and actinic keratoses (AKs). A limited resolution and the lack of visualization of cellular structures have hitherto restricted the use of OCT in the evaluation and differentiation of naevi and malignant melanoma $[8,9]$. Recently, however, speckle variance OCT has been introduced and made commercially available. The technology has been termed dynamic OCT (D-OCT). In addition to the images of traditional OCT scans, D-OCT allows the detection of blood flow in vivo, and visualizing the skin microvasculature. This advance in technology enables us to describe the vascular morphology better than hitherto in different skin diseases including, among others, cutaneous tumours and inflammatory skin diseases. A recent report has described the differences of vasculature in naevi and malignant melanoma [10], suggesting that D-OCT may aid in the early diagnosis of melanoma.

In this paper, we review the imaging of various skin pathologies with D-OCT. The depth of data varies significantly between the different disease entities studied, and in order to better explore the potential of this new technology the authors have opted for a broad approach and included preliminary data in the review.

\section{Technology}

OCT is a laser-based imaging modality that is capable of providing detailed pictures of subsurface tissue microstructure to a depth of $>1 \mathrm{~mm}$ in real time, and has been very successfully applied to imaging of the eye $[11,12]$. Multi-beam OCT uniquely provides images with lateral resolution $<7.5 \mu \mathrm{m}$, in real time, over the penetration depth of $>1 \mathrm{~mm}$, by combining the OCT data from multiple laser beams focused at slightly different depths in the tissue, scanned simultaneously through the region of interest [13]. This combination of high resolution and deep image penetration enabled the development of commercially available scanners utilizing multi-beam OCT which provides images revealing tissue morphology. Both vertical 'B scan' and horizontal 'en face' or 'C scan' images can be rapidly collected and displayed to the user for immediate interpretation.

'Standard' OCT relies on the small variations in intensity of back-scattered light from different tissue cellular microstructures to reveal the tissue morphology [14]. The resulting contrast between some tissue types can be quite low, and so there has been continued interest in the development of techniques with the potential of extracting further clinically useful information from the OCT data. One technique of great promise is D-OCT [15]. D-OCT involves rapidly repeating OCT scans, and analysing the statistics of the OCT signal, to detect regions of the OCT images which have changed between these successive scans. Most of the scanned tissue is unchanged, but any blood flow produces small changes in the D-OCT data that are detectable, thereby revealing the presence of blood vessels. The key technical challenge overcome in recent work is to remove, or compensate for, the effects of the 'bulk movement' of a hand-held OCT probe with respect to the patient's skin: even tiny movements of the probe threaten to 'wash out' the D-OCT signal from blood flow, but improvements to the D-OCT algorithms have largely overcome this problem.

In this paper a VivoSight ' $\mathrm{Rx}$ ' dermatological OCT scanner equipped with $20-\mathrm{kHz}$ swept-source laser and 'Dynamic OCT' processing and software (Michelson Diagnostics, Kent, www.vivosight.com) was used. The VivoSight OCT scanner has CE mark and FDA 510(k) for scanning external tissues of the human body, i.e. skin. The hand-held probe provides a $6 \mathrm{~mm} \times 6 \mathrm{~mm}$ field of view, and is easy to position on the lesion for the scanning process, which takes $10-20 \mathrm{~s}$. No contact gel is necessary. The device immediately provides vertical and horizontalplane OCT images, with a depth penetration of usually $1 \mathrm{~mm}$ or greater, for review by the user. The D-OCT data is collected simultaneously with the structural OCT data, and is immediately displayed on the structural OCT images as an overlay so that the relationship between the vessels and the structural image can be seen. Areas of motion are coloured red. Typically a 'stack' of 120 OCT images is captured for each lesion to form a 3-dimensional (3D) 'data block' (the density of images in the 3D block is configurable). The image stacks are exported as files in standard TIFF format for subsequent offline manipula- 
tion and analysis with the standard image processing tool ImageJ (available from NIH, imagej.nih.hiv/ij/). We also used proprietary software by Michelson Diagnostics and by the University of Modena for some of the statistical analyses and image rendering described in this article.

\section{D Imaging}

The en face view offers a visualization of the vascular network obtained by presenting the OCT scan in horizontal layers. In convex lesions this technique fails to capture the true organization of the vessels, since imaging does not adapt to the curved surface of the skin.

To better visualize the OCT scans, a $3 \mathrm{D}$ reconstruction technique is proposed that is able to show layers of the lesion at a constant depth from the outer skin surface. The $3 \mathrm{D}$ shape of the area of interest is presented to the clinician along with the underlying vascular network, reconstructed from the D-OCT data.

The first step of the method is to find the superficial surface of the lesion; the following algorithm describes the procedure:

1 Every slice is convolved with circular filters highlighting strong gradients

2 For each column of the slice the topmost pixel with strong gradient is chosen (by fixing a threshold $\mathrm{T}$ )

3 A median filter followed by a spline interpolation method smooths the curve for each slice; the same two methods are applied to obtain interslice smoothness For a 3D surface reconstruction, see figure 1 .

Once the surface has been computed, the lesion can be viewed in a depth-preserving en face view that highlights the depths of the detected vessels along the entire scan (fig. 2).

D-OCT allows detection of blood flow, and it is thus possible to extract a point cloud of all red points (easily obtained by a threshold on the red image channel).

The raw point cloud has two major problems: (i) it is composed of millions of points (up to 20 millions), and (ii) it has a high noise:signal ratio. We therefore subsampled the data taking half points for each direction (thus taking 1 point every 8 ), and we designed a filtering step to remove isolated red points (not sufficiently surrounded by other points). 3D Delaunay triangulation was then applied to obtain a solid mesh, followed by Laplacian smoothing for a better and more realistic presentation.

A $3 \mathrm{D}$ view of the vessels allows for immediate qualitative evaluation otherwise impossible with $2 \mathrm{D}$ visualization (fig. 3).

\section{Normal Skin}

The appearance of normal skin in OCT images varies according to regional differences, but the epidermis and the DEJ can always be reliably recognized. In the crosssectional OCT images of normal skin, a thin hyporeflective line is seen at the very top of the skin surface caused by the reflectivity of the skin [16]. Below this entrance signal, the epidermis is seen as a heterogeneous granular textured band of varying thickness. The stratum corneum can only be reliably visualized in glabrous skin where it appears as a thick hyporeflective layer with characteristic hyperreflective coiling structures representing eccrine sweat gland ducts [17]. The DEJ is seen as a marked change in contrast between the epidermis and the papillary dermis.

The normal skin is richly supplied with a vascular network forming two distinct horizontal plexuses in the dermis. The epidermis does not contain any vessels and therefore depends on diffusion from the dermal cells to meet its metabolic needs. The superficial horizontal vascular plexus gives rise to the arterial capillaries that form the dermal papillary loops that supply the epidermis [18]. D-OCT allows visualization of the vascular networks in the normal skin. Because of the varying thickness of the epidermis at different body locations, the vessels in the $\mathrm{D}$-OCT images come into view at different depths beneath the skin surface. In the cross-sectional D-OCT images, the dermal papillary loops can be recognized as small dots distributed in close proximity to the DEJ. In the en face view the D-OCT images demonstrate a welldefined and usually well-organized vascular pattern (fig. 4). Many factors, both intrinsic and extrinsic, influence the skin, and therefore the appearances of the vessels in D-OCT images vary at different body locations. In the en face view of normal facial skin, the diameters of the individual vessels appear larger, and the appearance of a cruder vascular network can be recognized compared to normal skin located on the arm or the leg (fig. 5). Independently of body location the vascular networks in normal skin appear well organized, and the individual vessels can usually be easily outlined in the en face D-OCT images. This is an important characteristic that may be used in distinguishing normal skin from lesional skin.

\section{Basal Cell Carcinoma}

BCC is the most common skin cancer, and the development of these tumours is closely linked to UV exposure. The lesions can histologically be divided into sub- 
Fig. 1.3D surface reconstruction for a sample scan.

Fig. 2. A comparison between the standard en face view and the depth-preserving en face view.

Fig. 3. A reconstructed vascular network.

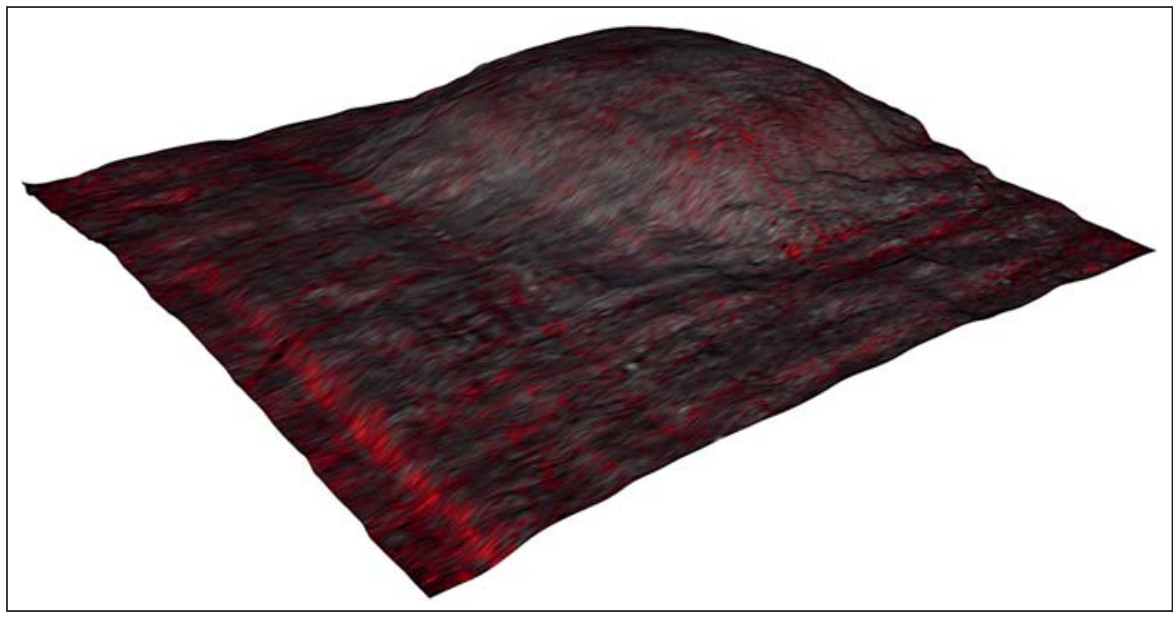

Standard en face view

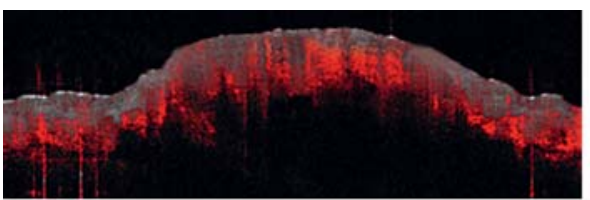

Depth-preserving en face view
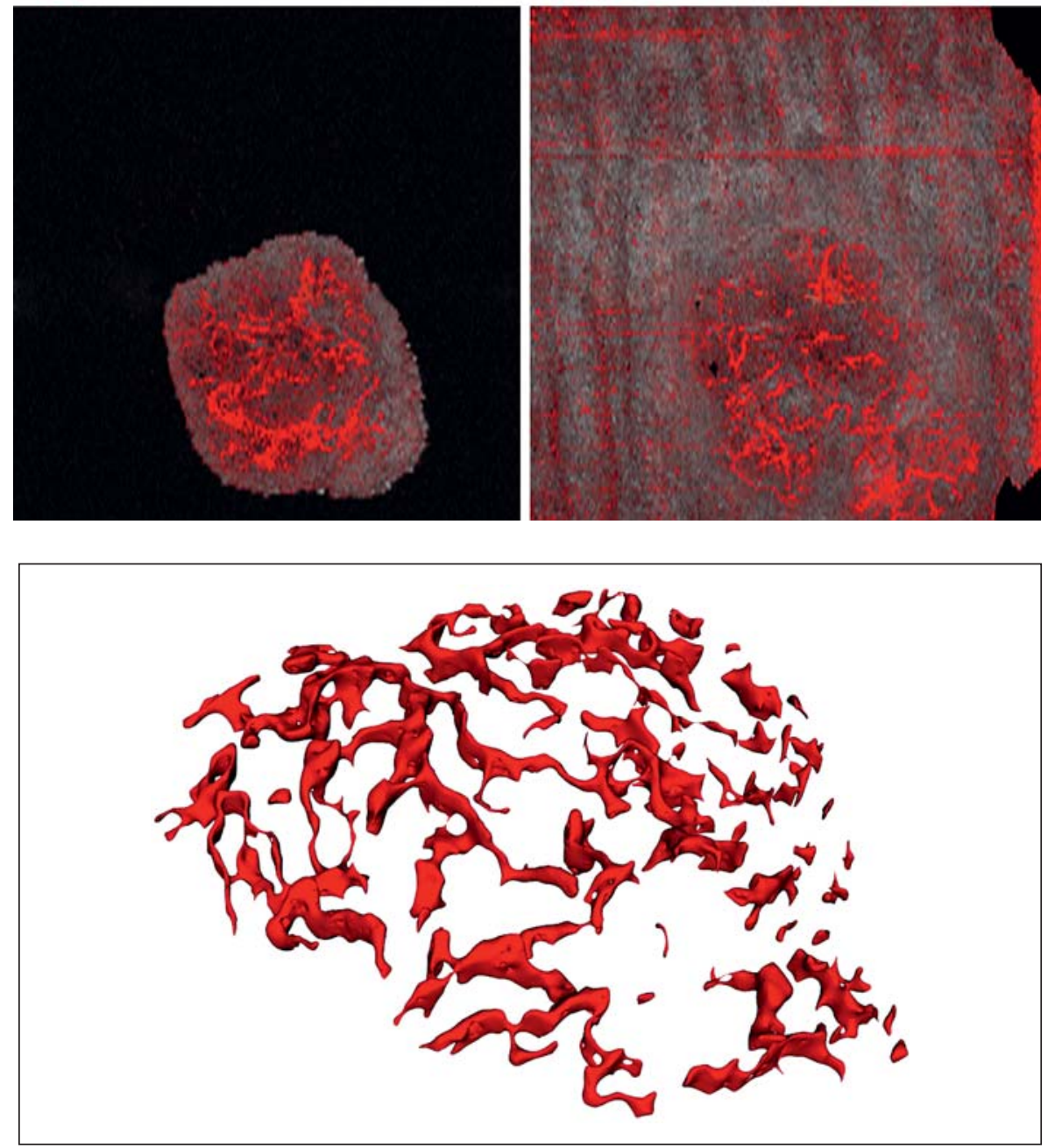

Dynamic OCT in Dermatology

Dermatology 2016;232:298-311 
4

Fig. 4. D-OCT en face view of normal skin located on the wrist of a 58-year-old patient (size: $6 \mathrm{~mm} \times 6 \mathrm{~mm}$ ). The image shown is at a skin depth of $0.44 \mathrm{~mm}$. The vessels appear well defined, and the vascular pattern is well organized.

Fig. 5. D-OCT en face view of normal skin located on the face of the same patient as in figure 4 (size: $6 \mathrm{~mm} \times 6 \mathrm{~mm}$ ). The image is at a skin depth of $0.44 \mathrm{~mm}$. The diameters of the vessels appear larger, and the network is cruder compared to the vessels in figure 4; however, the vascular pattern still appears well organized.
5

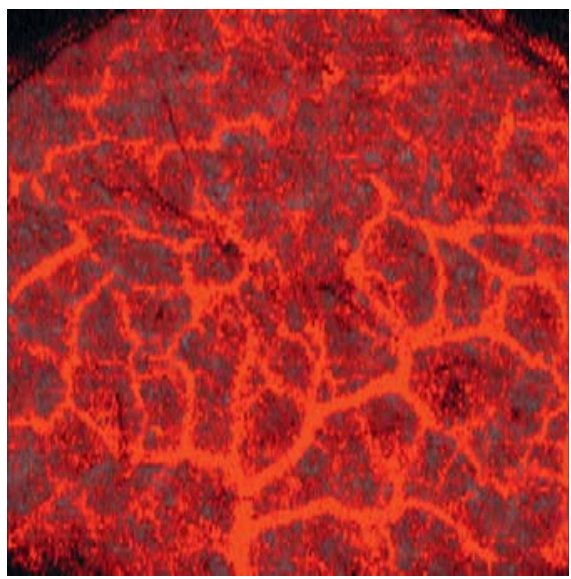

types associated with different risks of recurrence [19]. Clinically BCC can appear as pink patches, ulcerations, pearly papules/nodules or scar-like areas. They very rarely metastasize but are locally invasive and can become disfiguring if left untreated.

The major OCT characteristics of BCC lesions in OCT images include alteration of the DEJ, dark ovoid basal cell islands and often a hyporeflective peripheral boundary $[1,6]$. Combining major and minor OCT criteria for pattern analysis of the tissue enables diagnosis of BCC with a specificity of $\sim 75 \%$ and a sensitivity of $\sim 96 \%[2,7]$ and can also aid in the monitoring of medical treatments of BCC lesions [20-22]. An algorithm has been proposed for the subclassification of BCC using high-definition OCT but awaits further validation, while there is currently no data to suggest that standard OCT can reliably differentiate between BCC subtypes [5, 23-25].

Angiogenesis, the growth and expansion of the vasculature, is an important process in the growth and metastasis of many cancers, including NMSC [26]. The newly formed vessels supply the tumour with oxygen and nutrients required for continued growth, and typically angiogenesis is required for tumours to grow beyond 1-2 mm in size. The morphology of superficial vessels has long been an important feature in dermoscopic examination. The finding of arborizing vessels is a useful dermoscopic sign for the diagnosis of BCC [27], and in histopathology the finding of a loose and more vascularized dermis between tumour nests is a diagnostic clue for multifocal superficial BCC lesions. Thus, the potential benefit of in vivo imaging of the vasculature in BCCs seems promising.

D-OCT allows real-time visualization of the blood vessels within the BCC lesions with an imaging depth that enables detection of both superficial vessels and vessels in the deeper parts of the dermis. In BCC lesions, the D-OCT images demonstrate a change in the appearance of the overall pattern of vessel distribution. In the cross-sectional view of the superficial BCC type a progressive elongation of perpendicular vessel columns can be recognized. In the en face view of the lesions, the calibres of the blood vessels show variations, ranging from dilated, larger-thannormal vessels to the smallest detectable vessels. The arrangement of the vessels is disorganized with a multitude of minute vessels, losing the regularity seen in the uninvolved skin (fig. 6). D-OCT combines structural information about the lesion with information on vessel morphology and pattern. From this it is possible to recognize that the vessels are generally centred on the BCC lesions, and in some cases the vessels also appear to be infiltrating the edges of the tumour islands (fig. 7). The obvious differences in vasculature found by D-OCT suggest that this new technology may provide a significant improvement in non-invasive diagnosis and subtyping of BCC.

\section{AK and Squamous Cell Carcinoma}

AKs are characterized on OCT by a distinctive layering with a thickened, visible stratum corneum, a thickening of the epidermis and clear visibility of the DEJ. Different forms of stratum corneum disruption may be observed ranging from severe forms of compact hyperkeratotic scales with bright reflection to hyporeflective scales that corresponds to parakeratosis. On D-OCT imaging, AK shows a reticular network on en face view, which resembles the network of normal skin, but the vessels tend to form a larger calibre and a slightly irregular, broader network (fig. 8). 
6

Fig. 6. En face view of BCC located on the cheek (size: $6 \mathrm{~mm} \times 6 \mathrm{~mm}$ ). The image shows that the calibre of the blood vessels varies greatly. The vessels are arranged in a disorganized way with a multitude of minute vessels.

Fig. 7. En face view of a BCC located on the leg (size: $6 \mathrm{~mm} \times 6 \mathrm{~mm}$ ). The image shows a characteristic network of vessels lining the periphery of the tumour islands.

Fig. 8. D-OCT en face view of an AK located on the face showing a reticular network of vessels around the adnexal structures (size: $6 \mathrm{~mm} \times 6 \mathrm{~mm}$ ). In comparison to the vessels in the normal skin, these appear slightly more enlarged and less well organized as in normal skin.

Fig. 9. D-OCT en face view of Bowen's disease (SCC in situ) located on the trunk (size: $6 \mathrm{~mm} \times 6 \mathrm{~mm}$ ). In contrast to the vasculature in AK (fig. 8), the vessels are not arranged in a network but as predominantly dotted vessels.

Fig. 10. D-OCT en face view of an invasive SCC located on the forehead (size: $6 \mathrm{~mm} \times$ $6 \mathrm{~mm}$ ). The vessels are irregularly distributed and show marked differences in their calibre and morphology.

8

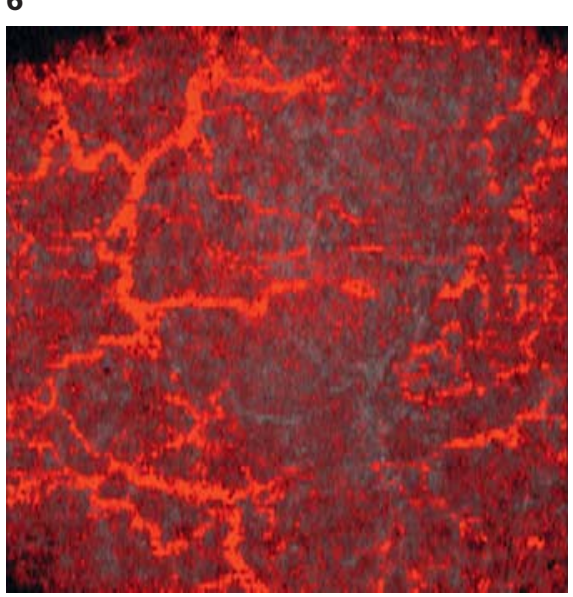

7

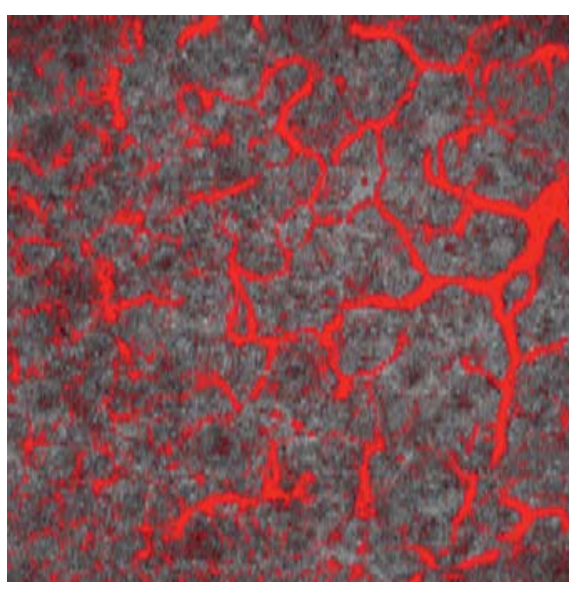

10

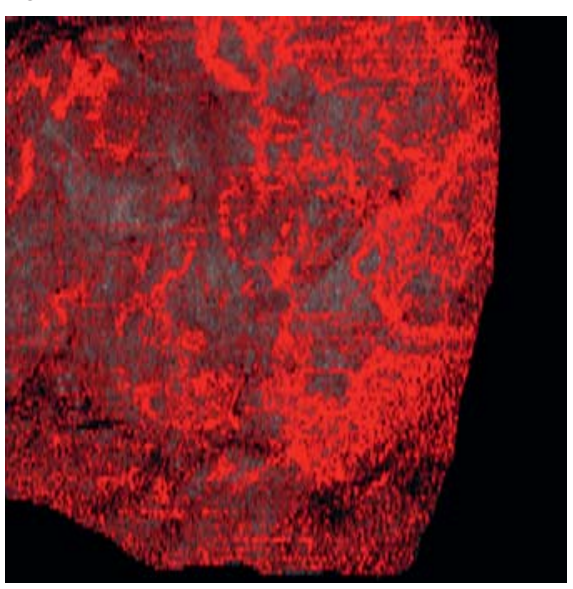

9
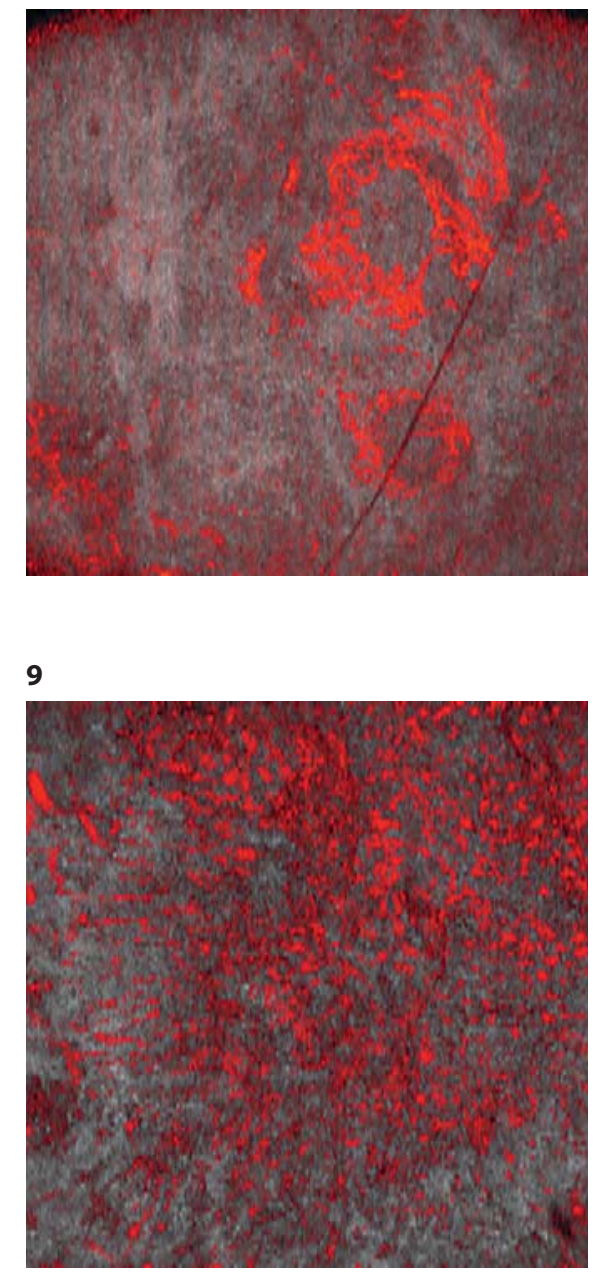
11

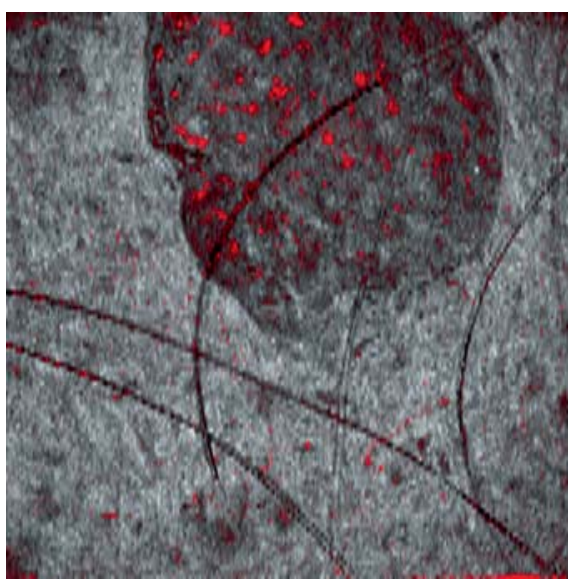

12

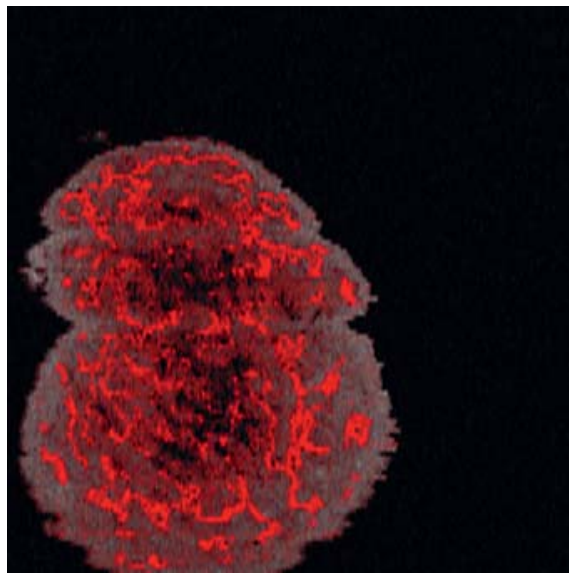

Fig. 11. D-OCT en face view of an irritated congenital naevus on the leg (size: $6 \mathrm{~mm} \times$ $6 \mathrm{~mm}$ ). The vessels are dotted and very regularly distributed within the lesion.

Fig. 12. D-OCT en face view of a nodular melanoma with the presence of long linear vessels in a chaotic distribution (size: $6 \mathrm{~mm}$ $\times 6 \mathrm{~mm})$.
The features of Bowen's disease/squamous cell carcinoma (SCC) in situ on structural, vertical OCT images are similar to those of AK. However, these lesions often tend to be less hyperkeratotic and show a marked thickening of the epidermis. By using D-OCT in these lesions, a significant difference in the vessel morphology is noted on en face view. In contrast to AK, the vessels in Bowen's disease/ SCC in situ are not arranged in a network but depending on the level of examination as dotted or semicircular or even in a fuzzy morphology (fig. 9). The dotted vessels seen in SCC in situ are larger than the capillaries observed in normal skin or psoriasis and are reliably found among SCC in situ independently of their body site location.

The differentiation of invasive SCC from in situ SCC or hyperkeratotic AK remains a challenge in OCT examination. The main problem is caused by hyperkeratotic scales, which impair the visualization of deeper structures and impede the clear visualization of the DEJ. Criteria for invasive SCC on OCT imaging include the loss of the typical layering (as visible in AK/SCC in situ), loss of clear demarcation of the DEJ and round or ovoid structures in the dermis of varying reflectivity. The vessel morphology on DOCT of SCC shows a diversified pattern of irregularly shaped and arranged vessels of different calibre (fig. 10).

\section{Naevi and Melanoma}

Melanocytic lesions are characterized by a variable vascular pattern according to their nature and dermal involvement. The vascular pattern in melanocytic lesions is better evaluated in horizontal sections or in $3 \mathrm{D}$ reconstructions.

Junctional naevi or naevi with a minimal dermal involvement show a vascular pattern usually comparable with the surrounding normal skin but sometimes more pronounced, displaying a pattern of regularly distributed dotted structures immediately below the epidermis. Only in the deep dermal component is it possible to identify a thin reticular architecture. In lesions presenting a conspicuous dermal component, larger vessels with an arcuate aspect, starting from the bottom of the lesion and directed toward the surface, are detectable in $3 \mathrm{D}$ reconstruction. The length of these arcuate figures is related with the tumour depth (the deeper, the longer), and they are regularly distributed throughout the lesion (fig. 11).

In melanoma, densely clustered red dots may be visible in the superficial dermis since the intra-epidermal proliferative phase. These dots present a chaotic distribution compared with naevi [9]. With the increase in tumour thickness, the red dots tend to aggregate in apparently linear structures. In invasive tumours with full papillary dermis involvement, usually long linear vessels are visible with angulated branches and irregular size. In thick invasive tumours, abundant irregular vessels are visible in the deep dermis, frequently presenting aneurysmal dilations and thin vertical structures directed towards the surface, resembling small spikes in $3 \mathrm{D}$ reconstruction (fig. 12).

\section{Inflammatory Conditions}

\section{Inflammatory and Degenerative Conditions}

Inflammatory skin diseases are associated with changes of the blood flow. Erythema is caused by a dilation of blood vessels, and chronic inflammation may affect the morphology of vessels such as change in their number, diameter and 3D architecture. 

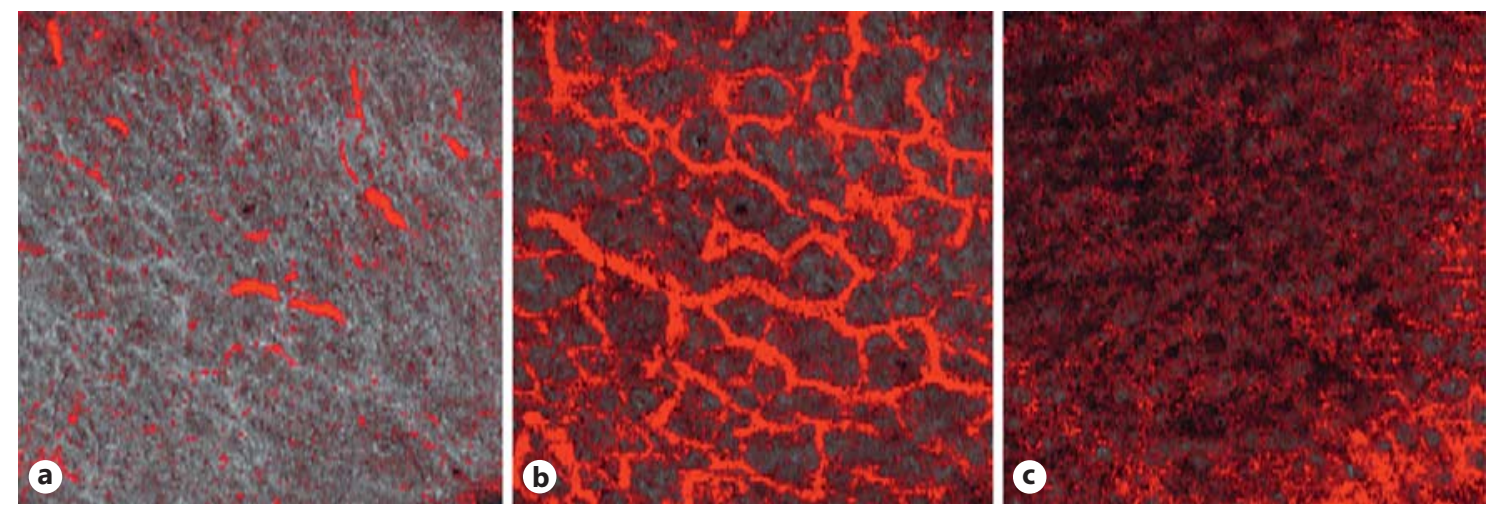

Fig. 13. Cheek of a rosacea patient (view size: $6 \mathrm{~mm} \times 6 \mathrm{~mm}$ en face), directly below the epidermis in the stratum papillare (a), stratum reticulare of the dermis (b) and same depth directly after dye laser treatment (c). The bright broad vessel network in rosacea disappeared completely.
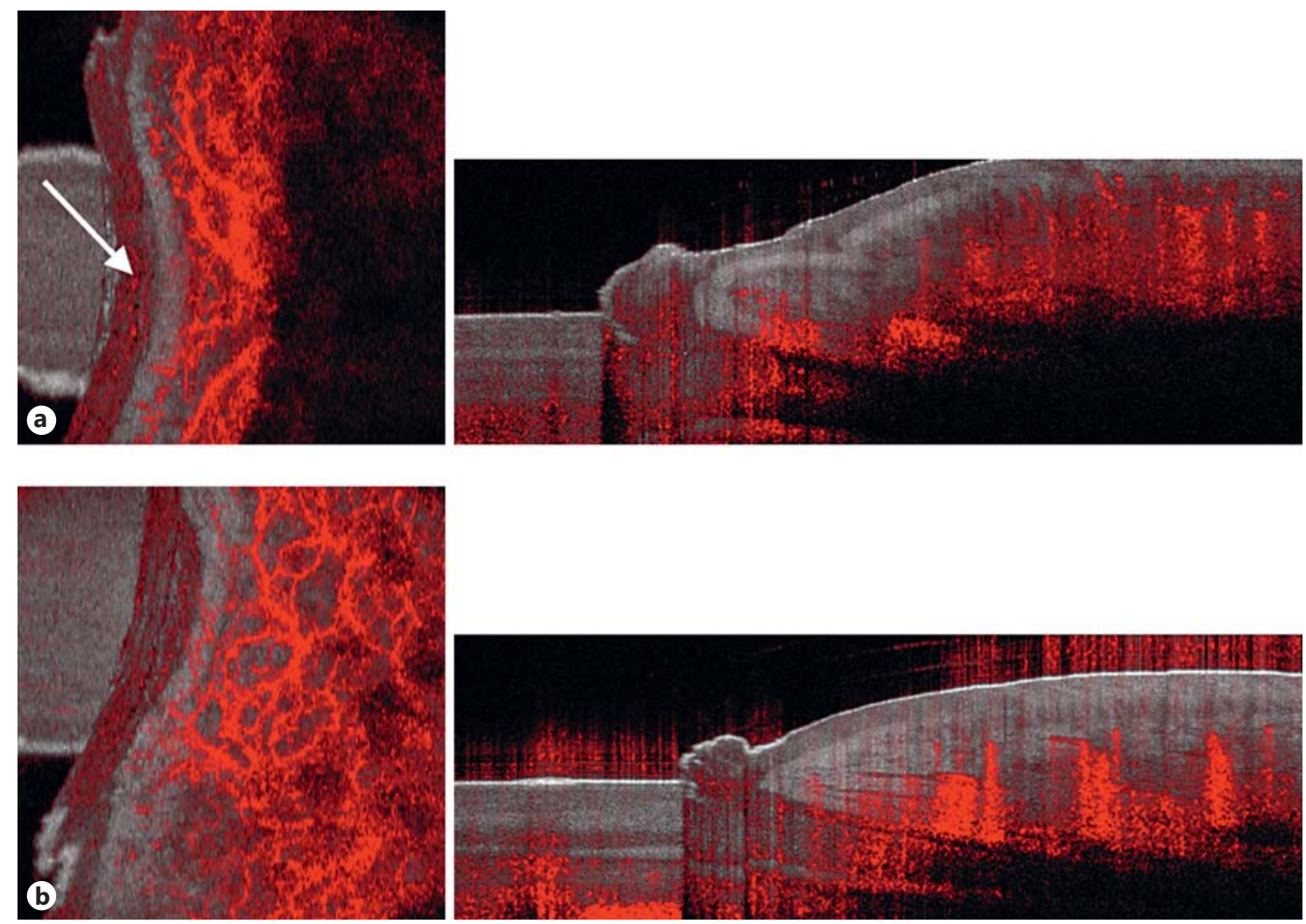

Fig. 14. Nail fold of a patient with late stage of scleroderma (a) compared to a healthy control person (b). In scleroderma, the epidermis and dermis are severely thickened. There is a rarefaction of the blood vessels. The dense network with small vessels is replaced by broader vessels with a Y-shaped branching. In the nail fold of the scleroderma patient, the number of vessels is increased (arrow). $6 \mathrm{~mm} \times 6 \mathrm{~mm}$ en face, $6 \mathrm{~mm} \times 2 \mathrm{~mm}$ transsectional.

\section{Rosacea}

In erythematous rosacea, thin, superficial blood vessels are dilated. This type can be influenced by topical application of brimonidine, an $\alpha_{2}$-adrenergic agonist, caus- ing a peripheral vasoconstriction of the superficial vessels $[28,29]$. When telangiectasias are present, also deeper vessels are affected which cannot be reached by topical treatment. In this case, dye laser therapy may be effective, 

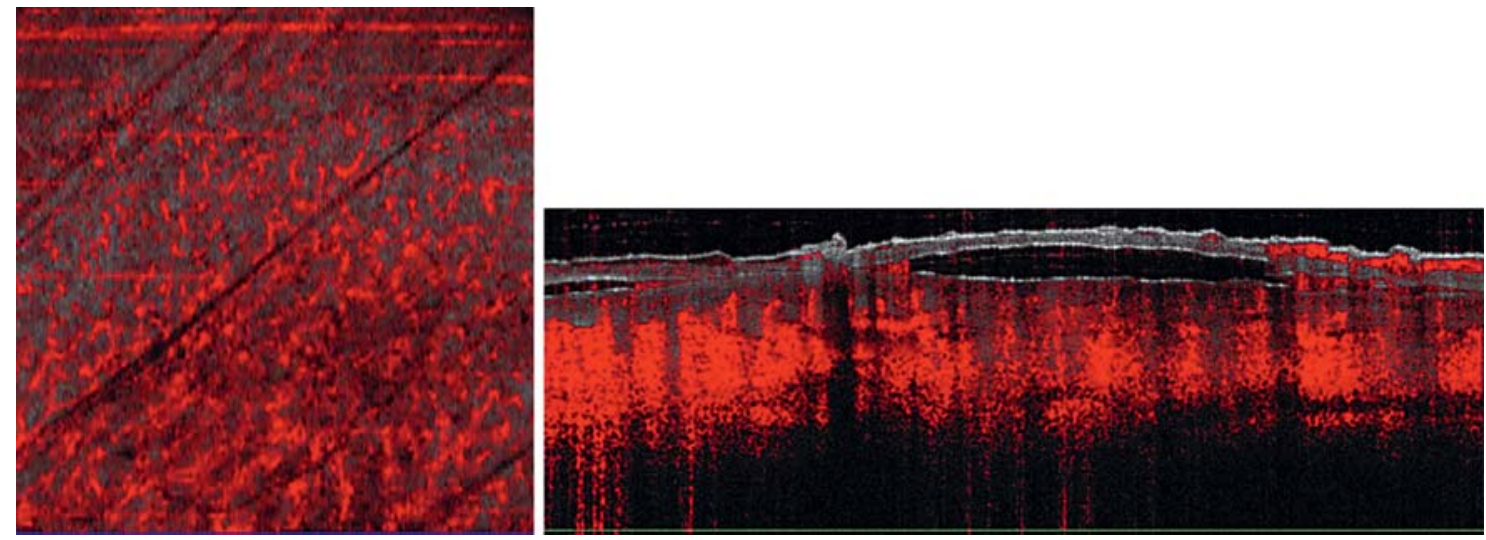

Fig. 15. Burn wound with subepidermal blister formation. At the bottom of the blister, blood vessels are visible, corresponding to a burn depth of grade IIa. $6 \mathrm{~mm} \times 6 \mathrm{~mm}$ en face, $6 \mathrm{~mm} \times 2 \mathrm{~mm}$ trans-sectional.

leading to coagulation of vessels. Coagulation of telangectasia following intense pulsed light treatment may be visualized using conventional OCT [30].

D-OCT allows an in vivo visualization of the blood vessels in rosacea. In superficial parts of the skin, it demonstrates a slightly increased number of small dotted vessels corresponding to erythema and in the deeper dermis a broader network (fig. 13a) corresponding to telangiectasia compared to persons without rosacea. Therapeutic effects of brimonidine as well as of laser treatment (fig. 13b, c) can be monitored and quantified over time using D-OCT.

\section{Scleroderma}

In systemic scleroderma, the dermis is thickened and stiff. After early inflammatory stages, the vasculature is affected [31-34]. The blood vessels are rarefied, causing necrosis of the fingertips due to ischaemia. On the other side, atypical loops of nail fold capillaries are a typical early sign of disturbed vessel architecture in scleroderma and provide an important diagnostic clue when recognised. Figure 14 shows the nail fold and fingertip of a patient with systemic scleroderma of the acral type, compared to a healthy control person.

\section{Wounds}

The differential diagnosis of chronic wounds and the assessment of burns are often complicated. While histological examination of the tissue may be necessary, it appears counterintuitive to biopsy when studying delayed wound healing. Furthermore, the study of a continuous process such as wound healing is not suited to repetitive biopsies as the same lesion can be studied only once in this manner. Therefore, a non-invasive diagnostic tool for imaging of wounds, especially of the blood vessels in a wound, is of great relevance $[35,36]$.

In addition, specific practical aspects may benefit from such imaging. In acute burn wounds, the determination of the wound depth due to thermal necrosis is of importance because it defines the choice of the therapeutic regime, i.e. conservative versus surgical treatment. OCT may be used to determine the burn depth [37-41]. DOCT may be of additional interest, because not only the depth of the thermolysed skin, but also the blood flow and coagulation of blood vessels can be imaged (fig. 15, 16).

In chronic venous insufficiency, the healthy-appearing unaffected skin of the lower leg shows dilated lymphatic vessels without flow (fig. 17a). In dermatosclerosis, deeper vessels are rarefied within a fibrotic and sclerotic tissue (fig. 17b). Additionally, the superficial vessels in the stratum papillare appear to exhibit a pathological morphology with clusters (fig. 17c). At the edge of the wound, the epithelium can be detected and monitored during regrowth (fig. 17d). The capillaries in the wound bed develop a glomerulus-like configuration with convoluted vessels.

\section{Scars}

Scar tissue differs from the adjacent healthy skin in clinical appearance (colour, texture, stiffness, surface contour), histological changes (epidermis, vasculature, collagen content, fibrosis, sclerosis, inflammation) and subjective features (pain, itch). Scars may be atrophic, hypertrophic or keloids. Subjective scores like the Vancouver scar scale have been established to assess scars, but 
Fig. 16. Burn wound with a superficial necrosis, 5 days after thermal injury (boiling water; view size: $6 \mathrm{~mm} \times 6 \mathrm{~mm}$ ). Increased number of blood vessels below the necrosis (a), corresponding to inflammation and wound healing, compared to healthy adjacent skin of the same depth (b). A burn depth of grade IIa was confirmed by OCT.
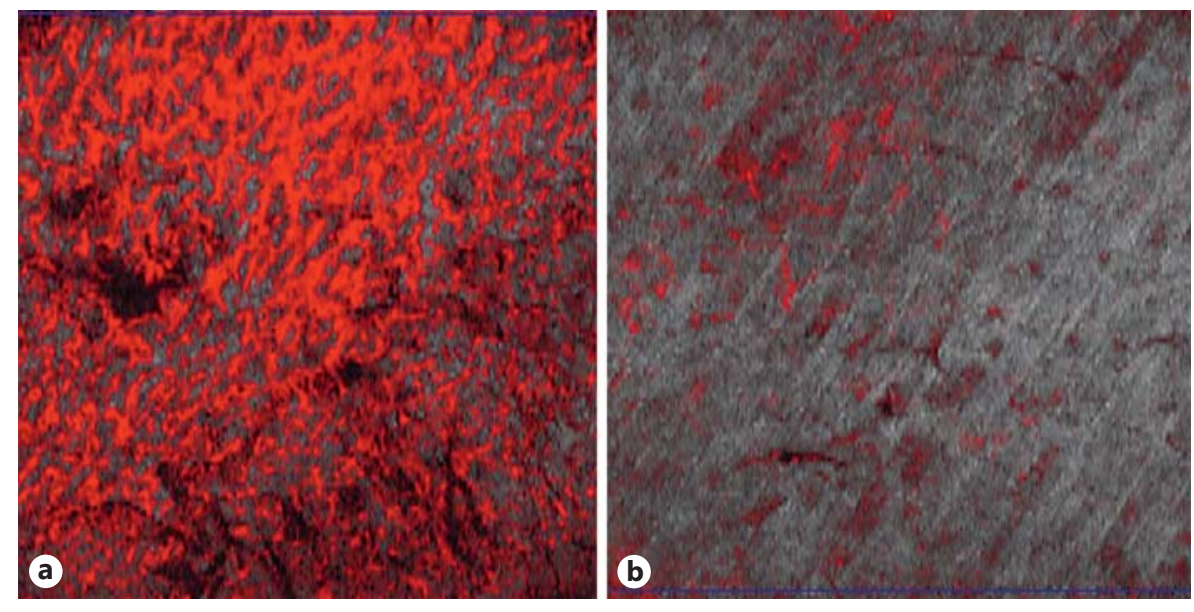

they lack objective quantitative data for more objective validation [42].

Scars after burn injury have been investigated using D-OCT. Gong et al. [42, 43] and Liew et al. [44] developed a speckle decorrelation OCT system and imaged scars [42-44]. They assessed the vasculature within the scars, the light attenuation coefficient of the tissue and the stiffness using OCT. Scars showed an increase in the number of blood vessels as well as disturbance in the vessel network compared to adjacent skin (fig. 18).

\section{Psoriasis}

Inflammation, epidermal proliferation and angiogenesis are important parts of the pathogenesis of psoriasis. Morphological changes of the blood vessels constitute an early sign of lesion evolution. Histologically, extended vessel loops in the papillary dermis are seen, while the epidermis typically shows acanthosis with severely elongated rete ridges in a regular pattern. D-OCT corresponds well to these morphological findings, showing spikes of small vessel loops in the stratum papillare (fig. 19).

\section{Discussion}

Although recent studies have demonstrated the applicability of OCT in the diagnosis of BCC and its mimickers, the wider use of OCT for other malignant and inflammatory diseases in dermatology is still limited. Morphological criteria of BCC and other NMSCs have been defined and proven useful for the diagnosis of these lesions. However, additional information on the vasculature of skin diseases adds more information and may further improve the diagnostic value of OCT technology. Especially in the field of skin neoplasms, the interaction between the tumour and its surrounding matrix is of major interest [4547]. It is well known that angiogenesis is increased in malignant tumours and may be associated with a higher risk of metastasis and mortality, and other technologies have therefore been applied for visualization of vessels in skin tumours [48]. Dermoscopy allows the evaluation of vessels and their patterns for different tumour types such as BCC, AK or melanoma, which have been well established, but it has a limited resolution and is restricted to the upper layers of the dermis $[49,50]$. Therefore, vascular changes have to be significant to be seen by dermoscopy. Reflectance confocal microscopy also allows the in vivo evaluation of vessels and suggests that the distinct vasculature of BCC as well as of AK and SCC in situ may well be visualized [51-54]. Reflectance confocal microscopy is however limited to the very superficial part of the papillary dermis, and it may be hypothesized that initial changes of the vascular pattern involved in neoplasia occur at deeper levels closer to the vascular plexus.

In contrast to existing techniques, D-OCT allows the visualization of vascular networks in the dermis up to a depth of 1.5-2 mm. The combination of vertical and en face view and the high number of scans (total of 120 scans in a $6 \times 6 \mathrm{~mm}$ area) add significant information. In this regard, the morphology of the single vessels, their arrangement within the lesions and the network which the vessels form within and around a tumour may be studied in detail. By using specifically designed software, 3D reconstruction of the vascular network is possible and adds further useful information. 

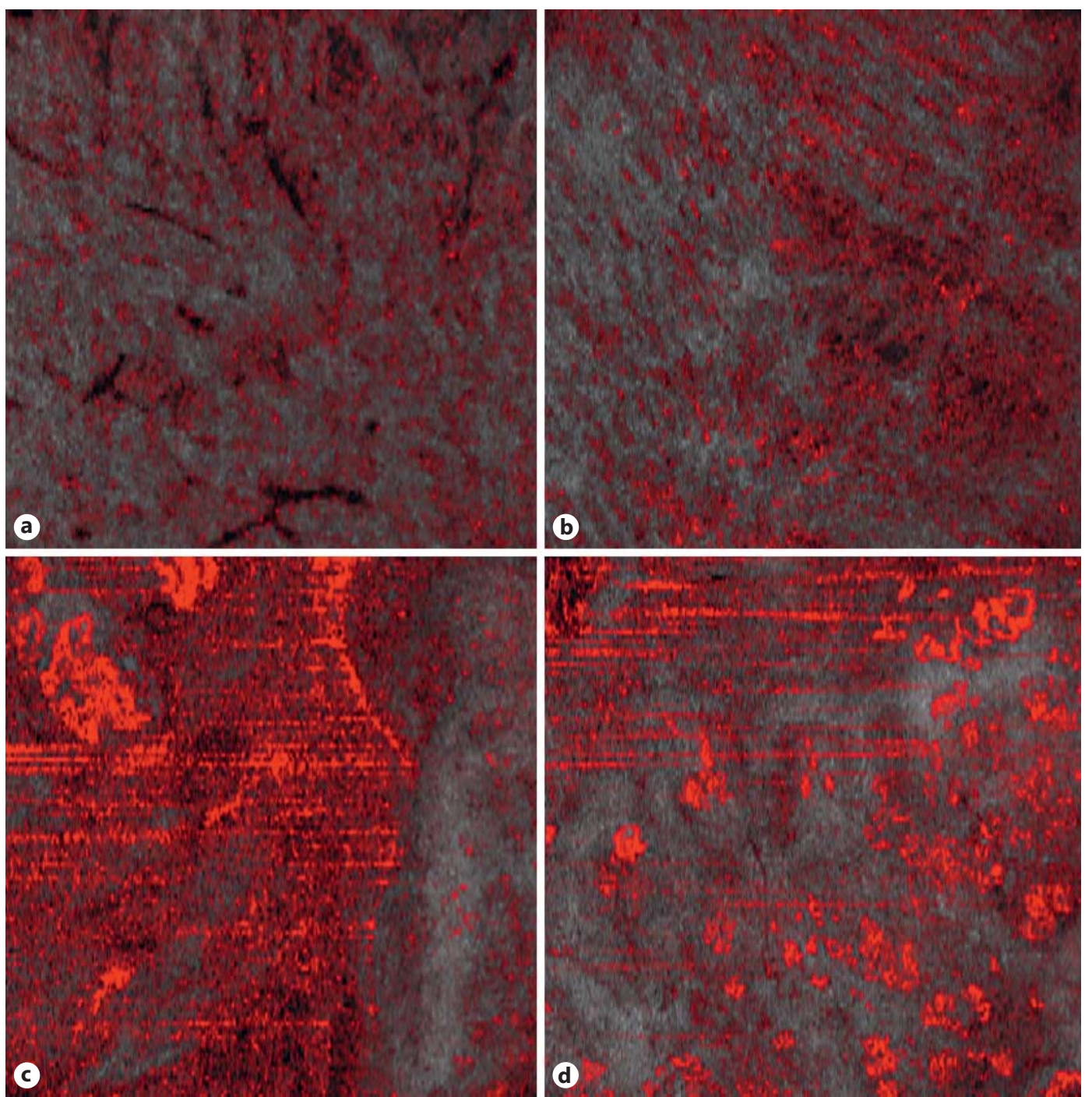

Fig. 17. Lower leg of a patient with a chronic venous ulcer. $6 \mathrm{~mm} \times 6 \mathrm{~mm}$ en face, $6 \times 2 \mathrm{~mm}$ trans-sectional. a Healthy-appearing skin outside the sclerotic area. Severely dilated lymphatic vessels without flow signal are seen. $\mathbf{b}$ Dermatosclerosis with slightly increased dotted vessels in the stratum papillare. $\mathbf{c}$ Border of the ulcer with bright larger dots of vessels. $\mathbf{d}$ Centre of the ulcer. The granulation tissue shows knots of blood vessels.

With regard to skin cancer, the additional information, which D-OCT provides, may be applied to improve diagnostic accuracy. For example, non-melanocytic skin tumours such as AK and superficial BCC apparently show significant differences in their vasculature. Whereas the vessels in AK are arranged in a network of vessels (fig. 8), superficial BCC is characterized by lining vessels surrounding the single tumour islands of BCC (fig. 7). Existing OCT systems have hitherto not shown good utility in differentiating between melanoma and benign naevi. Limited resolution of the technique inhibits the de- tection of early signs of malignancy, resulting in a low diagnostic accuracy - even for high-resolution OCT. In contrast, in vivo reflectance confocal microscopy increases the diagnostic accuracy in the diagnosis of melanoma and reduces the number of unnecessary biopsies, but only provides information about the most superficial parts of the tumours [48]. Pilot studies of D-OCT suggest differences in the vessel morphology and networks of naevi and melanoma. However, the results are preliminary and need to be confirmed by larger studies. 
Fig. 18. Scar on the cheek (view size: $6 \mathrm{~mm} \times 6 \mathrm{~mm}$ ). The vessels are increased in number and oriented in a parallel direction instead of a network pattern.

Fig. 19. Psoriasis (a) compared to healthy adjacent skin (b). Despite marked morphological differences (thickened and bright stratum corneum, acanthosis), the number of blood vessels is increased in psoriasis. Especially in the upper stratum papillare, loops of dilated capillaries are present (arrows). $6 \mathrm{~mm} \times 6 \mathrm{~mm}$ en face, $6 \mathrm{~mm} \times 2 \mathrm{~mm}$ trans-sectional.
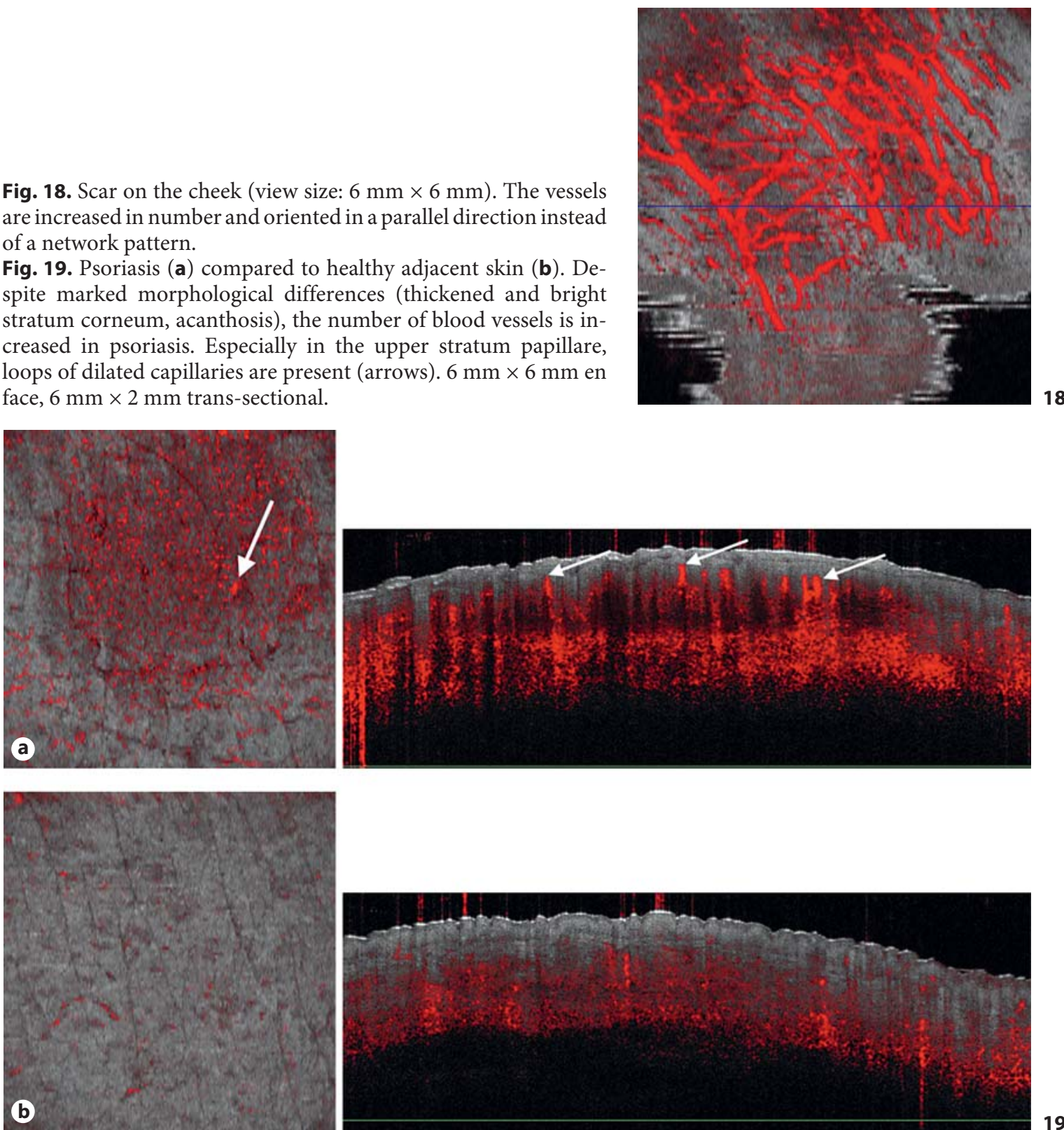

In addition to its use in the primary diagnosis of skin cancer, D-OCT may also be helpful to identify the individual tumour risk. It is well suggested that perivascular invasion of cutaneous SCC is associated with a greater malignant potential [55]. Assuming a tumour has a great increase in vascularity, this may therefore constitute a marker for a higher risk of metastasis. Existing methods, such as routine histology or specific immunohistology staining vessels, do not provide easily interpretable data on the vasculature of a skin tumour and are therefore difficult to compare with the situation in vivo. In conse- quence, vascularization of melanoma and NMSC is generally a poorly described risk factor. D-OCT may overcome these limitations of routine histology by enabling us to study the in vivo characterization of blood vessels and lymphatic vessels. D-OCT may therefore potentially help us to identify high-risk tumours, which may in consequence lead to better and more individualized treatments.

The microvasculature is affected by a number of diseases and a wider use of D-OCT is applicable in a variety of inflammatory skin diseases, chronic wounds and other areas of dermatological research. For example, the effects 
of certain therapies on vessels may be studied over time, e.g. intense pulsed light treatment or topical brimonidine in the treatment of rosacea $[29,30]$.

Overall, non-invasive technologies such as D-OCT provide the opportunity to evaluate dynamic changes in the skin over time and without interfering with the tissue, and therefore have the potential to provide data superior to sequential biopsies. In the future, a combination of different technologies may help to overcome the given limitations of the single technologies and give us new insights into the pathophysiology as well as the dynamic changes of skin diseases. This may not only help in a better understanding of skin pathology, but also in the development of new treatments.

\section{Acknowledgement}

This work is part of an EU-funded project (ADVANCE, Project No. 621015).

\section{Disclosure Statement}

M.U.: stakeholder in CMB Collegium Medicum Berlin GmbH; clinical trials for Almirall, Biofrontera, Leo Pharma and Novartis; paid lectures for Almirall, Biofrontera, Galderma, Leo Pharma, Mavig GmbH, Michelson Diagnostics. J.H. and R.W. are employees of Michelson Diagnostics.

\section{References}

1 Welzel J, Lankenau E, Birngurber R, Engelhardt R: Optical coherence tomography of the human skin. J Am Acad Dermatol 1997;37: 958-963.

2 Mogensen M, Joergensen TM, Nuernberg BM, et al: Assessment of optical coherence tomography imaging in the diagnosis of nonmelanoma skin cancer and benign lesions versus normal skin: observer-blinded evaluation by dermatologists and pathologists. Dermatol Surg 2009;35:965-972.

3 Olmedo JM, Warschaw KE, Schmitt JM, et al: Optical coherence tomography for the characterization of basal cell carcinoma in vivo: a pilot study. J Am Acad Dermatol 2006;55:408-412.

4 Coleman AJ, Richardson TJ, Orchard G, et al: Histological correlates of optical coherence tomography in non-melanoma skin cancer. Skin Res Technol 2013;19:10-19.

5 Maier T, Braun-Falco M, Hinz T, et al: Morphology of basal cell carcinoma in high definition optical coherence tomography: en-face and slice imaging mode, and comparison with histology. J Eur Acad Dermatol Venereol 2013;27:97-104.

6 Hussain AA, Themstrup L, Jemec GB: Optical coherence tomography in the diagnosis of basal cell carcinoma. Arch Dermatol Res 2015;307:1-10.

7 Ulrich $\mathrm{M}$, von Braunmuehl T, Kurzen H, Dirschka T, Kellner C, Sattler E, Berking C, Welzel J, Reinhold U: The sensitivity and specificity of optical coherence tomography for the assisted diagnosis of nonpigmented basal cell carcinoma: an observational study. Br J Dermatol 2015;173:428-435.

8 Gambichler T, Plura I, Schmid-Wendtner M, Valavanis K, Kulichova D, Stücker M, Pljakic A, Berking C, Maier T: High-definition optical coherence tomography of melanocytic skin lesions. J Biophotonics 2015;8:681-686.
\9 Boone MA, Norrenberg S, Jemec GB, Del Marmol V: High-definition optical coherence tomography imaging of melanocytic lesions: a pilot study. Arch Dermatol Res 2014;306: $11-26$.

10 De Carvalho N, Ciardo S, Cesinaro A, Jemec G, Ulrich M, Welzel J, Holmes J, Pellacani G: In vivo micro-angiography by means of speckle-variance optical coherence tomography (SV-OCT) is able to detect microscopic vascular changes in naevus to melanoma transition. J Eur Acad Dermatol Venereol 2015, Epub ahead of print.

$\checkmark 11$ Huang D, et al: Optical coherence tomography. Science 1991;254:1178-1181.

12 Bouma B, Tearney C: Handbook of Optical Coherence Tomography. New York, Taylor \& Francis, 2001.

13 Holmes J, Hattersley S, Stone N, et al: Multichannel Fourier domain OCT system with superior lateral resolution for biomedical applications. Proc SPIE. Bellingham, SPIE, 2008, vol 6847: Coherence domain optical methods and optical coherence tomography XII, $\mathrm{p}$ $68470 \mathrm{O}$.

14 Huang D, Swanson EA, Lin CP, Schuman JS, Stinson WG, Chang W, Hee MR, Flotte T, Gregory K, Puliafito CA: Optical coherence tomography. Science 1991;254:1178-1181.

15 Mariampillai A, Standish BA, Moriyama EH, Khurana M, Munce N, Leung MKK, Jiang J, Cable A, Wilson BC, Vitkin IA, Yang VXD: Speckle variance detection of microvasculature using swept-source optical coherence tomography. Optics Lett 2008;33:1530-1532.

16 Welzel J: Optical coherence tomography in dermatology: a review. Skin Res Technol 2001;7:1-9.

17 Mogensen M, Morsy HA, Thrane L, Jemec GB: Morphology and epidermal thickness of normal skin imaged by optical coherence tomography. Dermatology 2008;217:14-20.
18 Braverman IM: The cutaneous microcirculation. J Invest Dermatol 2000;5:3-9.

19 Strutton GM: Pathological variants of basal cell carcinoma. Australas J Dermatol 1997; 38(suppl 1):S31-S5.

20 Mogensen M, Thrane L, Joergensen TM, Andersen PE, Jemec GB: Optical coherence tomography for imaging of skin and skin diseases. Semin Cutan Med Surg 2009;28:196-202.

21 Themstrup L, Banzhaf CA, Mogensen M, Jemec GB: Optical coherence tomography imaging of non-melanoma skin cancer undergoing photodynamic therapy reveals subclinical residual lesions. Photodiagn Photodyn Ther 2014;11:7-12.

22 Banzhaf CA, Themstrup L, Ring HC, Mogensen M, Jemec GB: Optical coherence tomography imaging of non-melanoma skin cancer undergoing imiquimod therapy. Skin Res Technol 2014;20:170-176.

-23 Boone MA, Suppa M, Pellacani G, Marneffe A, Miyamoto M, Alarcon I, Ruini C, Hofmann-Wellenhof R, Malvehy J, Jemec GB, Del Marmol V: High-definition optical coherence tomography algorithm for discrimination of basal cell carcinoma from clinical BCC imitators and differentiation between common subtypes. J Eur Acad Dermatol Venereol 2015;29:1771-1780.

24 Boone MA, Norrenberg S, Jemec GB, Del Marmol V: Imaging of basal cell carcinoma by high-definition optical coherence tomography: histomorphological correlation. A pilot study. Br J Dermatol 2012;167:856-864.

25 Gambichler T, Plura I, Kampilafkos P, Valavanis K, Sand M, Bechara FG, Stücker M: Histopathological correlates of basal cell carcino$\mathrm{ma}$ in the slice and en face imaging modes of high-definition optical coherence tomography. Br J Dermatol 2014;170:1358-1361. 
-26 Johnson KE, Wilgus TA: Multiple roles for VEGF in non-melanoma skin cancer: angiogenesis and beyond. J Skin Cancer 2012;2012: 483439.

27 Argenziano G, Zalaudek I, Corona R, Sera F, Cicale L, Petrillo G, et al: Vascular structures in skin tumors: a dermoscopy study. Arch Dermatol 2004;140:1485-1489.

-28 Urban J, Siripunvarapon AH, Meekings A, Kalowitz A, Markowitz O: Optical coherence tomography imaging of erythematotelangiectatic rosacea during treatment with brimonidine topical gel $0.33 \%$ : a potential method for treatment outcome assessment. J Drugs Dermatol 2014;13:821-826.

-29 Themstrup L, Ciardo S, Manfredi M, Ulrich M, Pellacani G, Welzel J, Jemec GB: In vivo, micro-morphological vascular changes induced by topical brimonidine studied by dynamic optical coherence tomography. J Eur Acad Derm Venereol 2016, Epub ahead of print.

-30 Ring HC, Mogensen M, Banzhaf C, Themstrup L, Jemec GB: Optical coherence tomography imaging of telangiectasias during intense pulsed light treatment: a potential tool for rapid outcome assessment. Arch Dermatol Res 2013;305:299-303.

- 31 Abignano G, Aydin SZ, Castillo-Gallego C, Liakouli V, Woods D, Meekings A, Wakefield RJ, McGonagle DG, Emery P, Del Galdo F: Virtual skin biopsy by optical coherence tomography: the first quantitative imaging biomarker for scleroderma. Ann Rheum Dis 2013;72:1845-1851.

- 32 Babalola O, Mamalis A, Lev-Tov H, Jagdeo J: Optical coherence tomography (OCT) of collagen in normal skin and skin fibrosis. Arch Dermatol Res 2014;306:1-9.

-33 Su P, Cao T, Tang MB, Tey HL: In vivo highdefinition optical coherence tomography: a bedside diagnostic aid for morphea. JAMA Dermatol 2015;151:234-235.

- 34 Ring HC, Mogensen M, Hussain AA, Steadman N, Banzhaf C, Themstrup L, Jemec GB: Imaging of collagen deposition disorders using optical coherence tomography. J Eur Acad Dermatol Venereol 2015;29:890-898.

35 Paul DW, Ghassemi P, Ramella-Roman JC, Prindeze NJ, Moffatt LT, Alkhalil A, Shupp JW: Noninvasive imaging technologies for cutaneous wound assessment: a review. Wound Repair Regen 2015;23:149-162.
36 Kuck M, Strese H, Alawi SA, Meinke MC, Fluhr JW, Burbach GJ, Krah M, Sterry W, Lademann J: Evaluation of optical coherence tomography as a non-invasive diagnostic tool in cutaneous wound healing. Skin Res Technol 2014;20:1-7.

37 Kim KH, Pierce MC, Maguluri G, Park BH, Yoon SJ, Lydon M, Sheridan R, de Boer JF: In vivo imaging of human burn injuries with polarization-sensitive optical coherence tomography. J Biomed Opt 2012;17:066012.

38 Todorović M, Jiao S, Ai J, Pereda-Cubián D, Stoica G, Wang LV: In vivo burn imaging using Mueller optical coherence tomography. Opt Express 2008;16:10279-10284.

39 Kim KH, Pierce MC, Maguluri G, Park BH, Yoon SJ, Lydon M, Sheridan R, de Boer JF: In vivo imaging of human burn injuries with polarization-sensitive optical coherence tomography. J Biomed Opt 2012;17:066012.

40 Srinivas SM, de Boer JF, Park H, Keikhanzadeh K, Huang HE, Zhang J, Jung WQ, Chen Z, Nelson JS: Determination of burn depth by polarization-sensitive optical coherence tomography. J Biomed Opt 2004;9:207-212.

41 Park BH, Saxer C, Srinivas SM, Nelson JS, de Boer JF: In vivo burn depth determination by high-speed fiber-based polarization sensitive optical coherence tomography. J Biomed Opt 2001;6:474-479.

42 Gong P, Chin L, Es'haghian S, Liew YM, Wood FM, Sampson DD, McLaughlin RA: Imaging of skin birefringence for human scar assessment using polarization-sensitive optical coherence tomography aided by vascular masking. J Biomed Opt 2014;19:126014.

43 Gong P, McLaughlin RA, Liew YM, Munro PR, Wood FM, Sampson DD: Assessment of human burn scars with optical coherence tomography by imaging the attenuation coefficient of tissue after vascular masking. J Biomed Opt 2014;19:21111.

44 Liew YM, McLaughlin RA, Gong P, Wood FM, Sampson DD: In vivo assessment of human burn scars through automated quantification of vascularity using optical coherence tomography. J Biomed Opt 2013;18:061213.

45 Desch A, Strozyk EA, Bauer AT, Huck V, Niemeyer V, Wieland T, Schneider SW: Highly invasive melanoma cells activate the vascular endothelium via an MMP-2/integrin av 35 induced secretion of VEGF-A. Am J Pathol 2012;181:693-705.

46 Schoenfeld J, Jinushi M, Nakazaki Y, Wiener D, Park J, Soiffer R, Neuberg D, Mihm M, Hodi FS, Dranoff G: Active immunotherapy induces antibody responses that target tumor angiogenesis. Cancer Res 2010;70:1015010160.
47 Weis SM, Cheresh DA: Tumor angiogenesis: molecular pathways and therapeutic targets. Nat Med 2011;17:1359-1370.

48 Mogensen M, Jemec GB: Diagnosis of nonmelanoma skin cancer/keratinocyte carcinoma: a review of diagnostic accuracy of nonmelanoma skin cancer diagnostic tests and technologies. Dermatol Surg 2007;33:11587114 .

49 Zalaudek I, Kreusch J, Giacomel J, Ferrara G, Catricalà C, Argenziano G: How to diagnose nonpigmented skin tumors: a review of vascular structures seen with dermoscopy. II. Nonmelanocytic skin tumors. J Am Acad Dermatol 2010;63:377-386.

50 Zalaudek I, Kreusch J, Giacomel J, Ferrara G, Catricalà C, Argenziano G: How to diagnose nonpigmented skin tumors: a review of vascular structures seen with dermoscopy. I. Melanocytic skin tumors. J Am Acad Dermatol 2010;63:361-374.

51 Longo C, Lallas A, Kyrgidis A, Rabinovitz H, Moscarella E, Ciardo S, Zalaudek I, Oliviero M, Losi A, Gonzalez S, Guitera P, Piana S, Argenziano G, Pellacani G: Classifying distinct basal cell carcinoma subtype by means of dermatoscopy and reflectance confocal microscopy. J Am Acad Dermatol 2014;71:716-724. e1.

52 Rishpon A, Kim N, Scope A, Porges L, Oliviero MC, Braun RP, Marghoob AA, Fox CA, Rabinovitz HS: Reflectance confocal microscopy criteria for squamous cell carcinomas and actinic keratoses. Arch Dermatol 2009; 145:766-772.

53 Ulrich M, Kanitakis J, González S, LangeAsschenfeldt S, Stockfleth E, Roewert-Huber J: Evaluation of Bowen disease by in vivo reflectance confocal microscopy. Br J Dermatol 2012;166:451-453.

54 Pellacani G, Pepe P, Casari A, Longo C: Reflectance confocal microscopy as a secondlevel examination in skin oncology improves diagnostic accuracy and saves unnecessary excisions: a longitudinal prospective study. $\mathrm{Br}$ J Dermatol 2014;171:1044-1051.

55 Breuninger H, Schaumburg-Lever G, Holzschuh J, Horny HP: Desmoplastic squamous cell carcinoma of skin and vermilion surface: a highly malignant subtype of skin cancer. Cancer 1997;79:915-919. 